\title{
The LHCb Silicon Inner Tracker
}

P. Sievers Physik-Institut University of Zürich

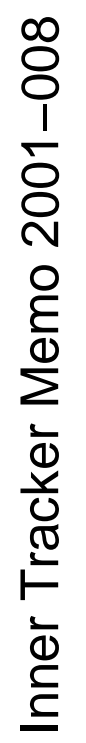

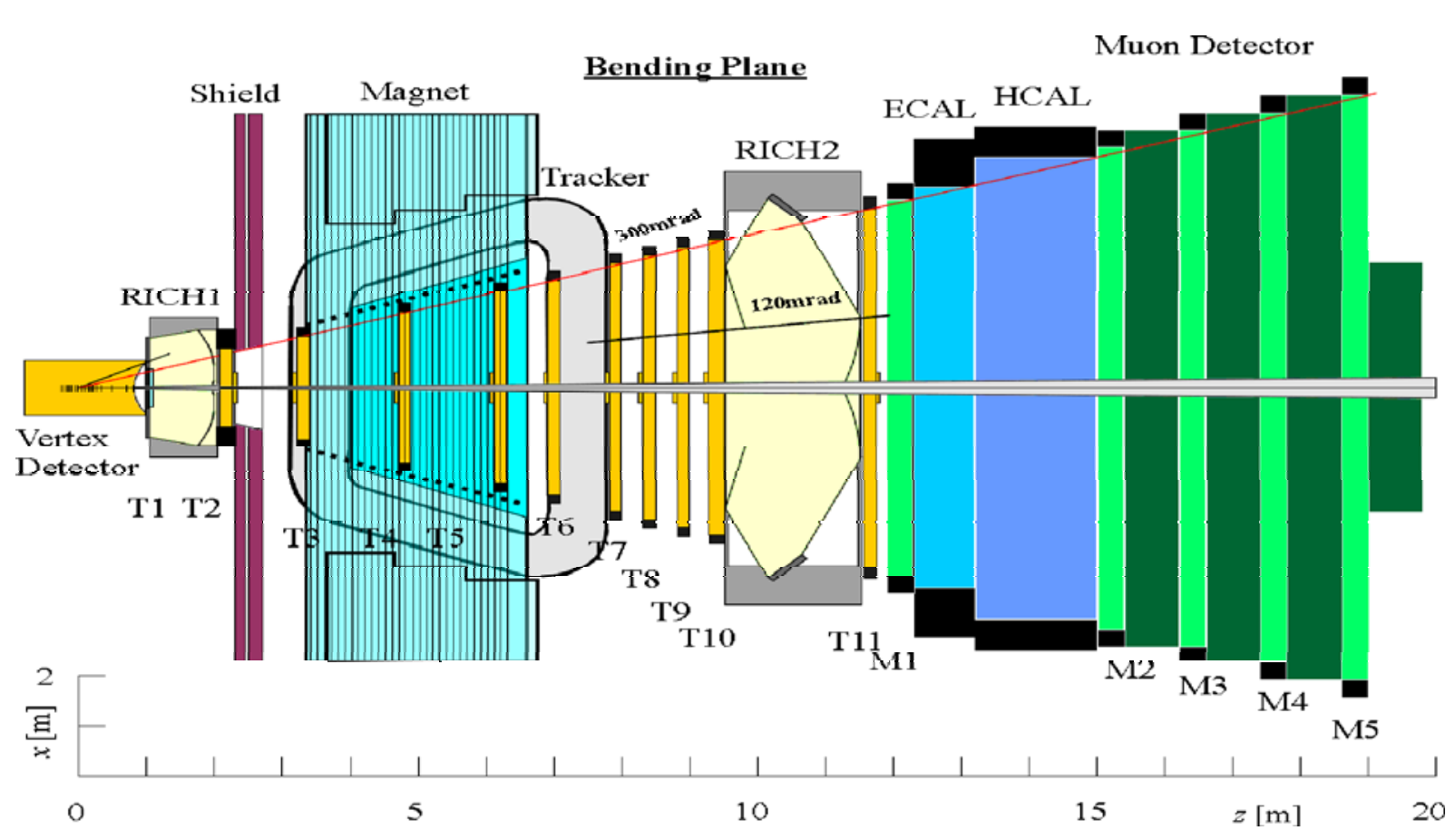

http://lhcb-track.web.cern.ch/lhcb-track/ 


\section{Requirements for the LHCb Inner Tracker}

Q very good momentum resolution $(\Delta p / p \approx 3 \%$ ) limited by multiple scattering $\longrightarrow$ material budget moderate spacial resolution $(100 \mu \mathrm{m})$

O particle rates up to $10^{4} \mathrm{~mm}^{-2} \mathrm{~s}^{-1}$

C occupancy has to stay low (few percent)

C signal shaping time of $25 \mathrm{~ns}$

silicon is adopted as technology for the LHCb Inner Tracker 


\section{Challenges}

(2 large pitch sensors reduce number of readout channels (costs)

( long sensor ladders $\mathrm{S} / \mathrm{N}$-performance

O small w/p S/N-performance

full charge collection efficiency 


\section{Station Layout}

Q nine stations

P four layers each

(2 vertical strips with small stereoview

- $22 \mathrm{~cm}$ long ladders

(2) $14 \mathrm{~m}^{2}$ of single sided silicon

beampipe is conical!

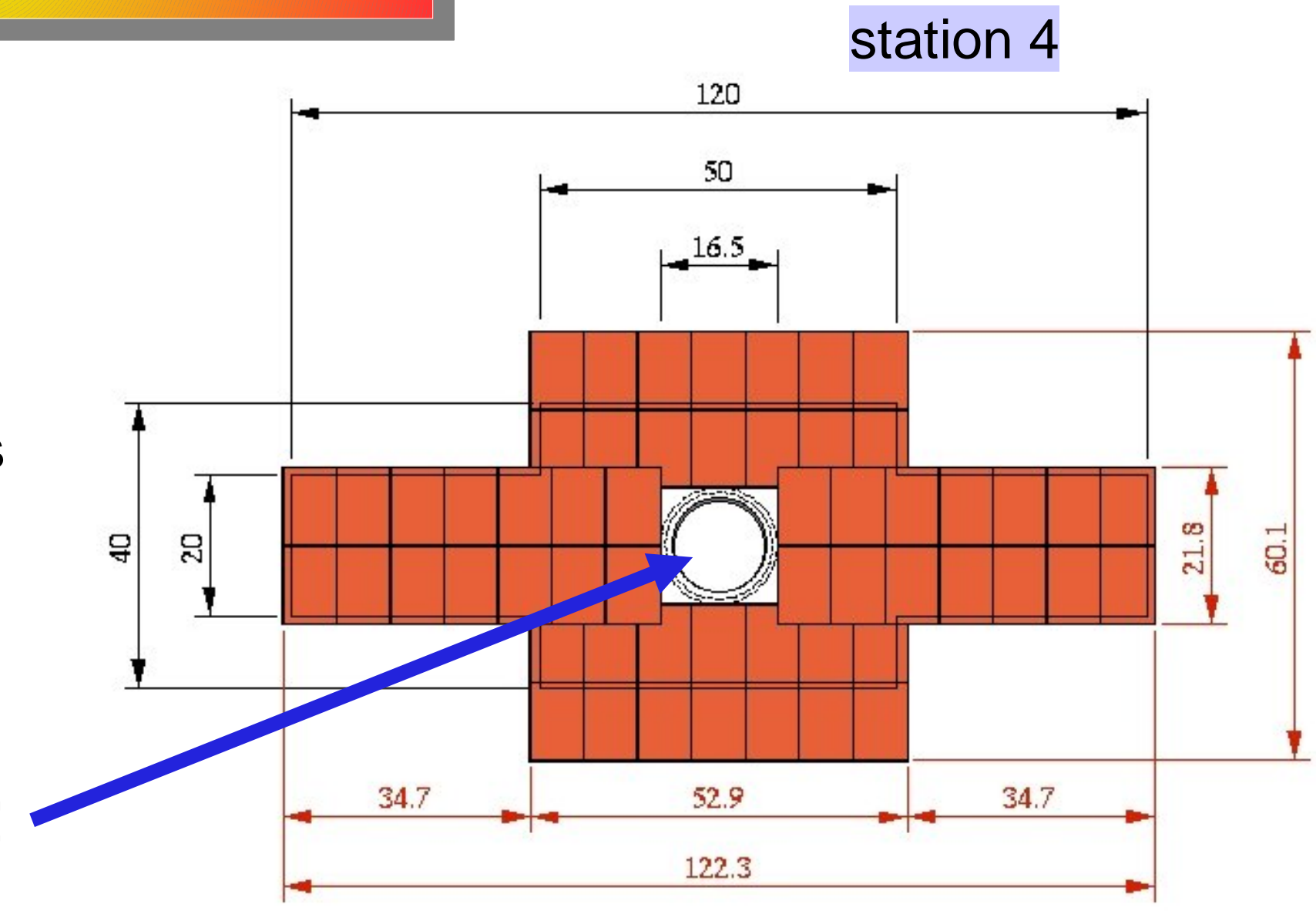

layout of every station is different

4 standard and independent boxes 


\section{Sensor Layout and Ladder Design}

Q sensor produced from 6" wafers

(2 length physical: $110 \mathrm{~mm}$ sensitive: $108 \mathrm{~mm}$

(2) width physical: $78 \mathrm{~mm}$ sensitive: $76 \mathrm{~mm}$

(2 strip pitch $237.5 \mu \mathrm{m}$ 320 strips
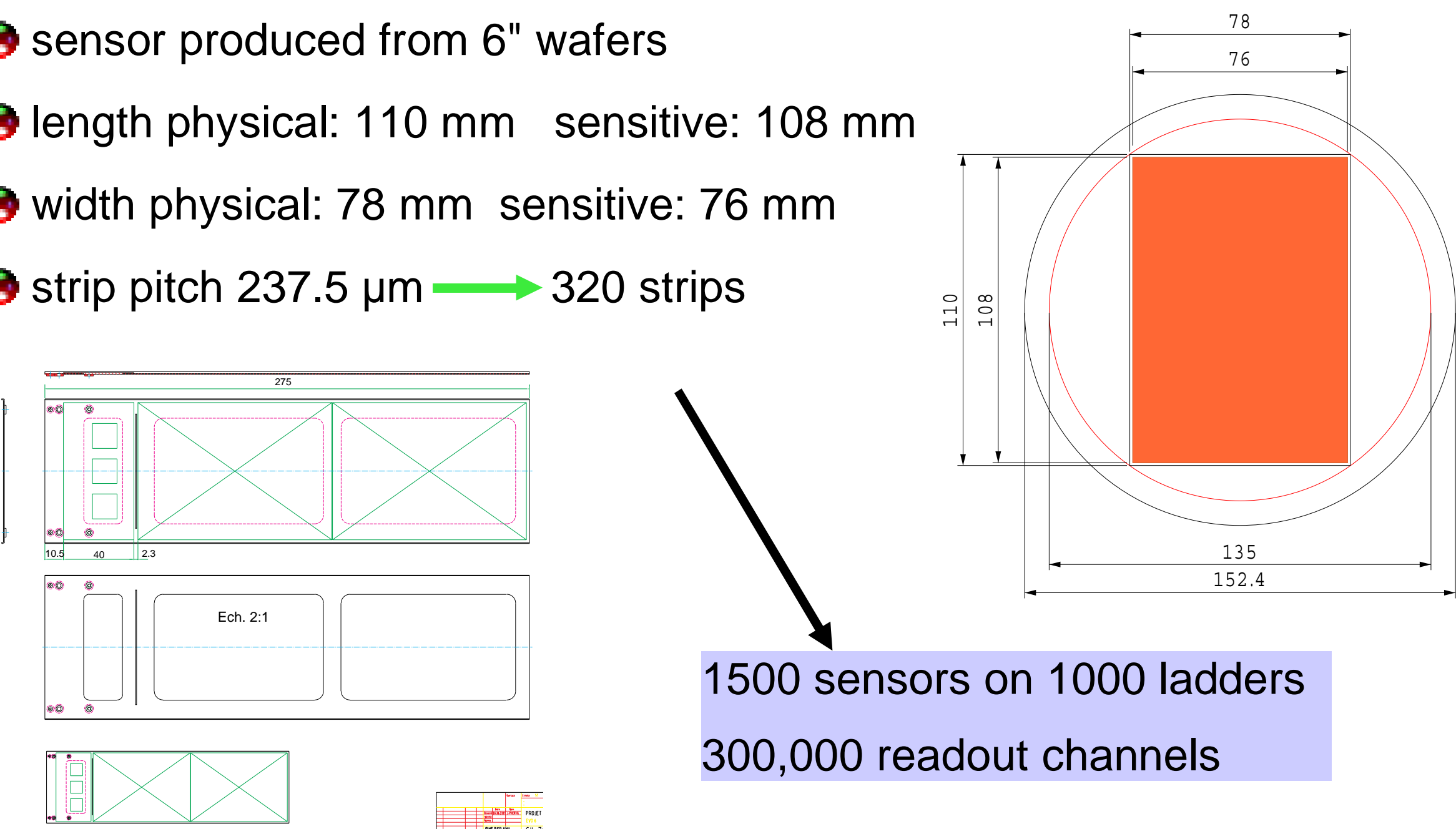

1500 sensors on 1000 ladders 300,000 readout channels 


\section{Multi-geometry prototype sensors from} company SPA Detector (Kiev)

(2p+ strips on n-type substrate

(2) pitch of $240 \mu \mathrm{m}$, single sided

( thickness of $300 \mu \mathrm{m}$

( oxygentated 4" wafers

(2 AC-coupled, polysilicon biased

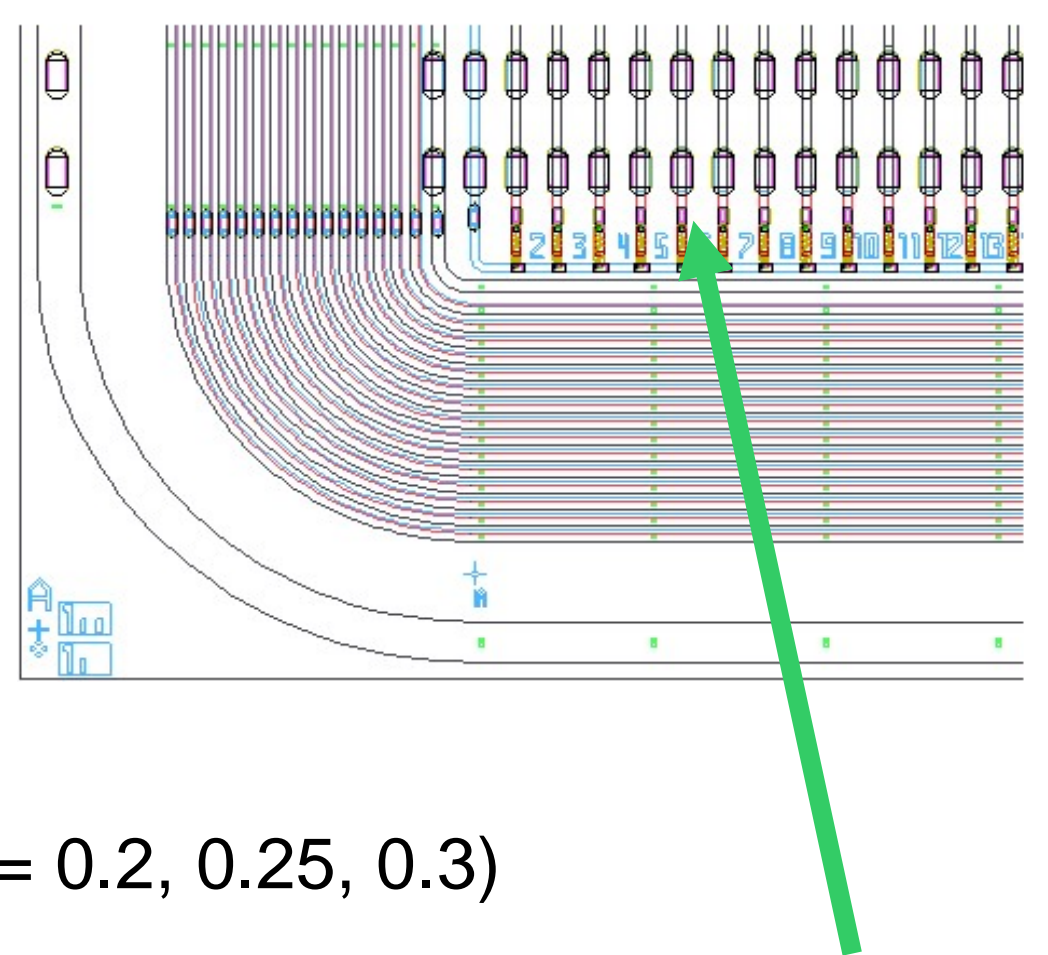

(2 two types: over- and under metallized

64 strips $66.6 \mathrm{~mm}$ long 


\section{Characterization of prototype sensors}

(2 leakage current

$\boldsymbol{\theta}$ total strip capacitance
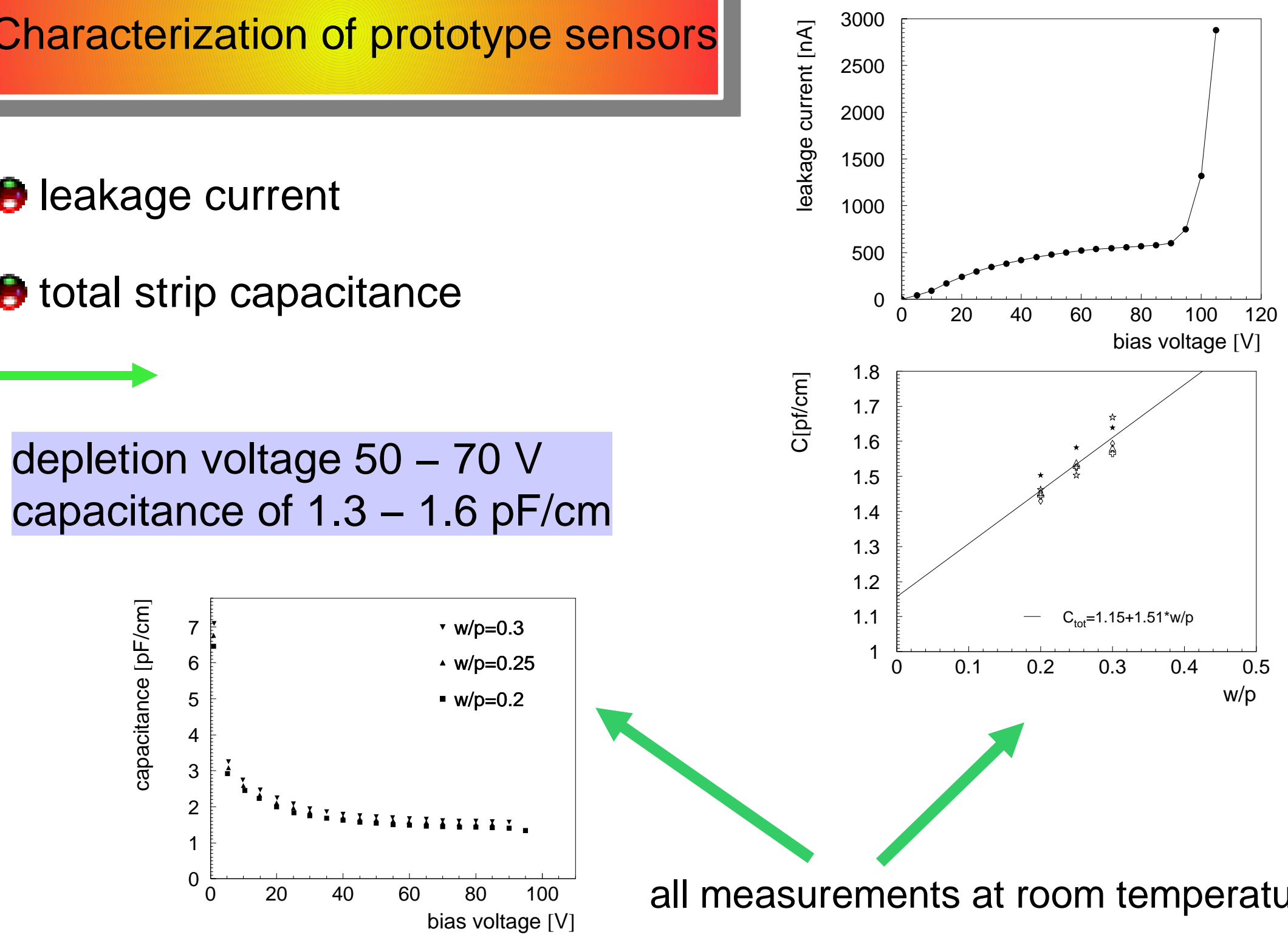

all measurements at room temperature 


\section{Laboratory Measurements}

๑ readout chip HELIX128

C measurements with Laser and Ru106

$\boldsymbol{\theta}$ also test of irradiated sensors

$$
\eta \text {-function }
$$

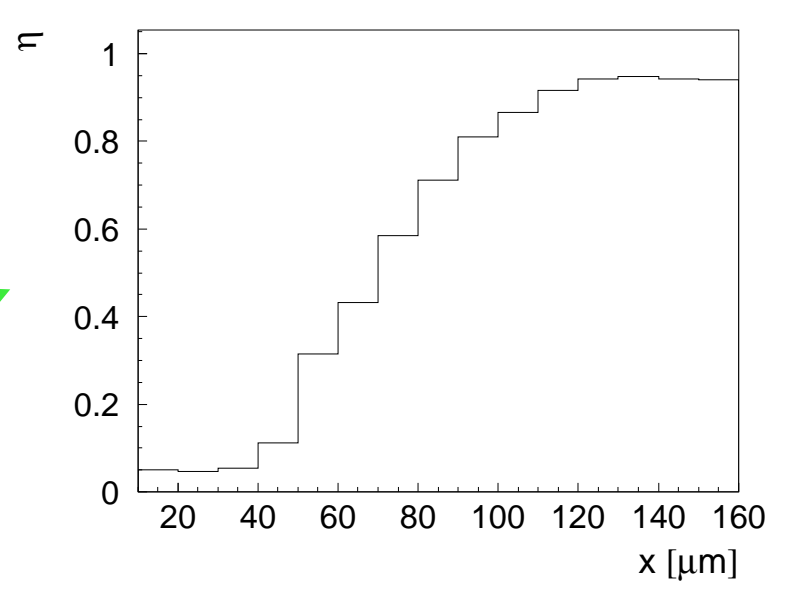

\section{$\mathrm{S} / \mathrm{N}$ degrades with higher w/p}

Irradiation corresponding 10 years of operation

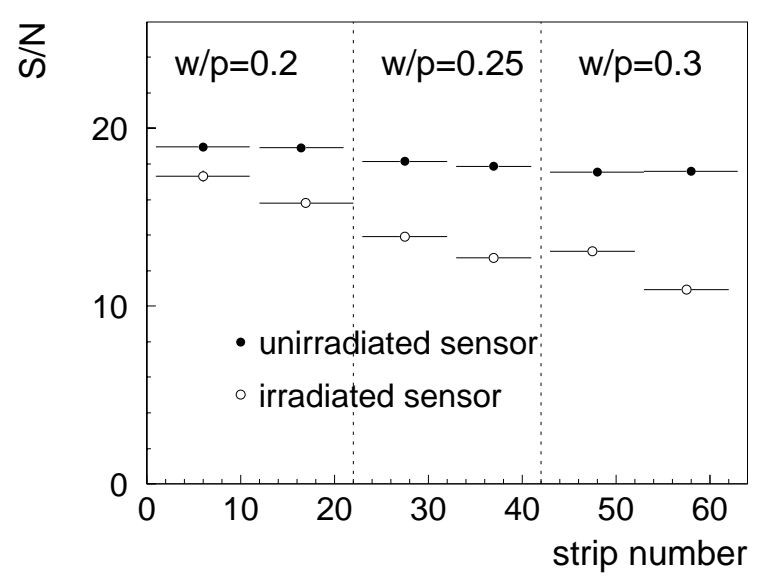




\section{Testbeam in May 2001}

measure resolution and efficiency $\&$ get some experience

(2) T7 test facility at CERN (9 GeV pions)

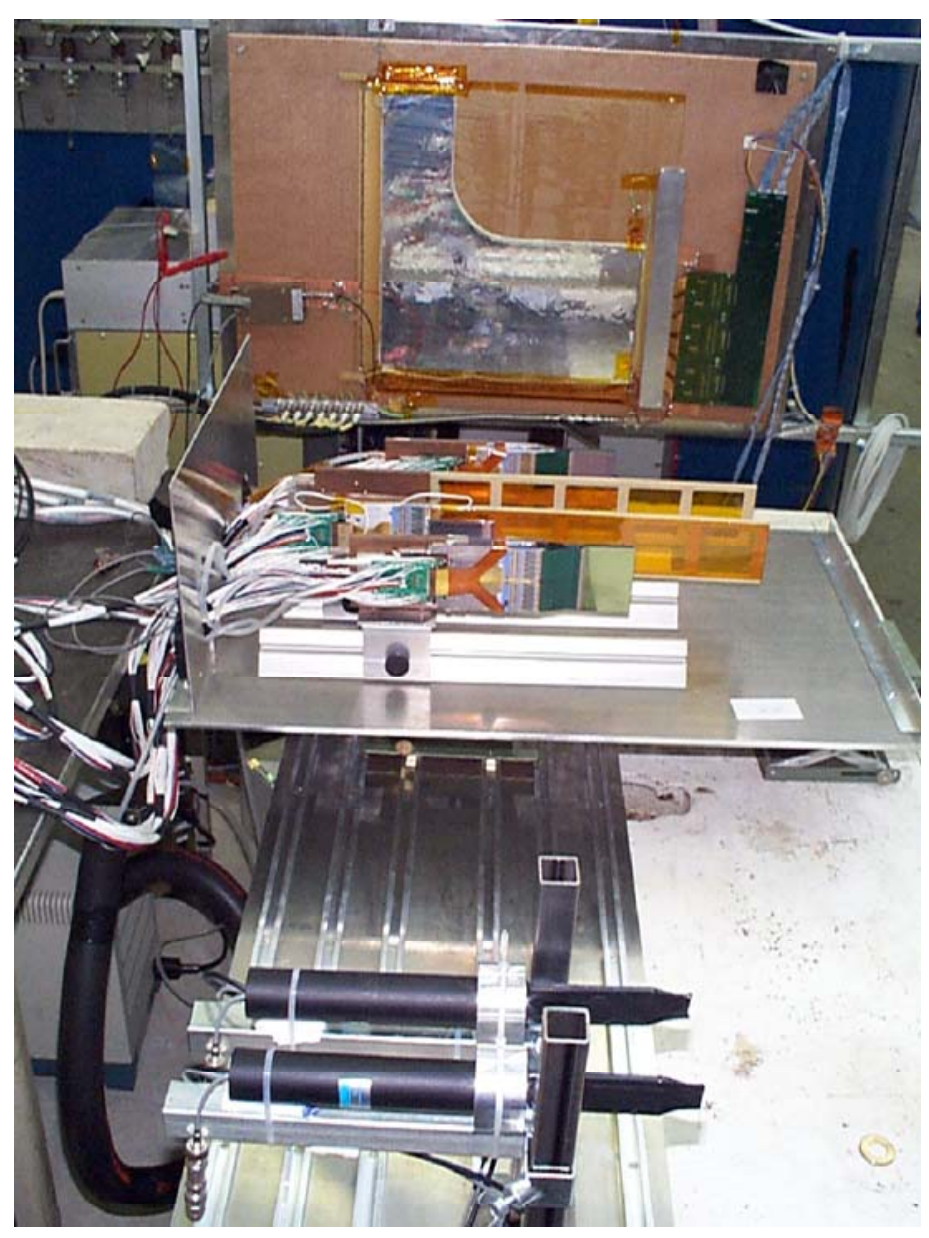

(2) used complete readout system of HERA-B Vertex detector

Q two ladders and beam telescope 


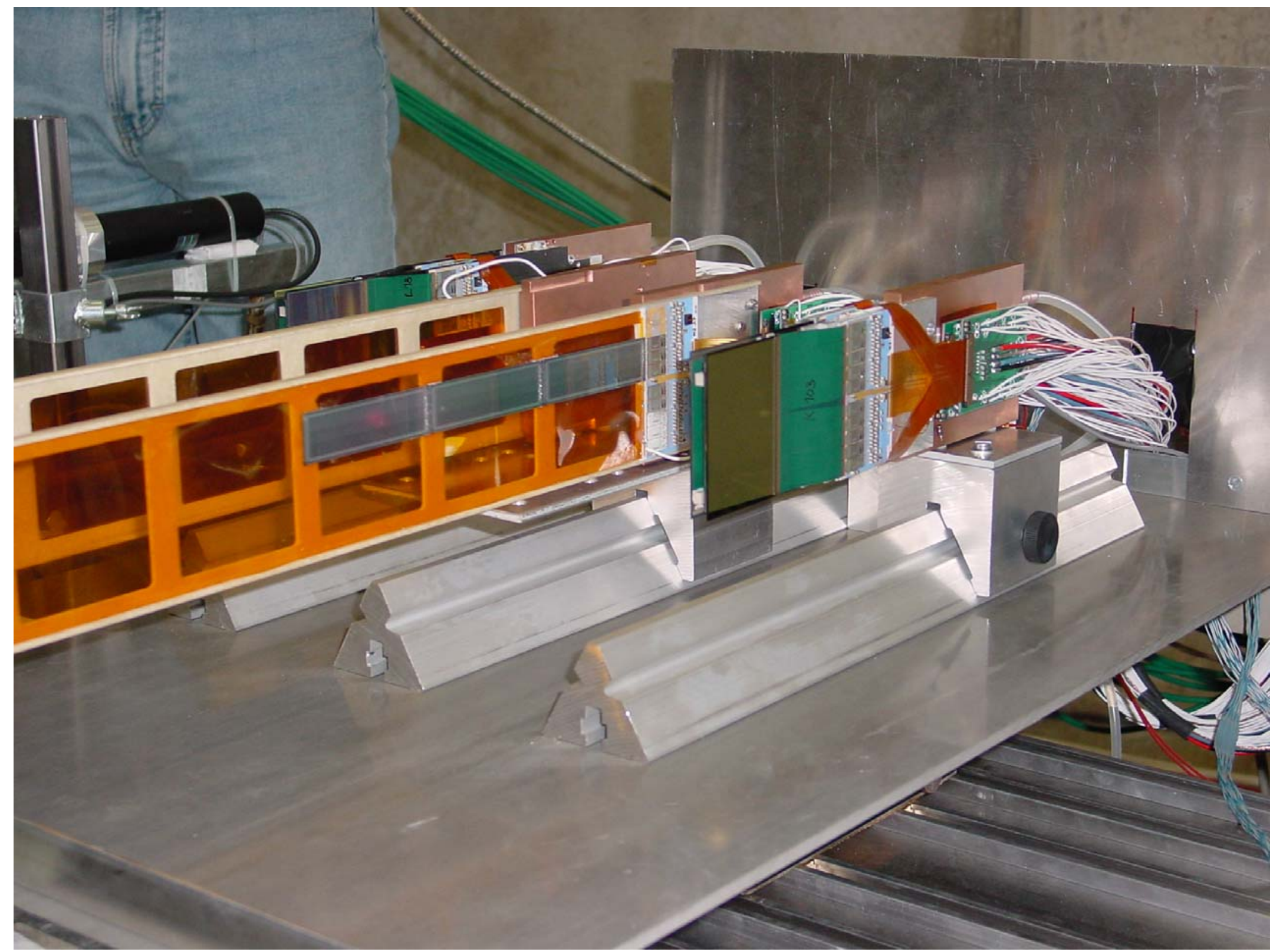




\section{Priliminary Testbeam Results for Short Sensor Ladder}

$\mathrm{S} / \mathrm{N}=20.3 / 19.9 / 19.4$ for different $w / p$ regions

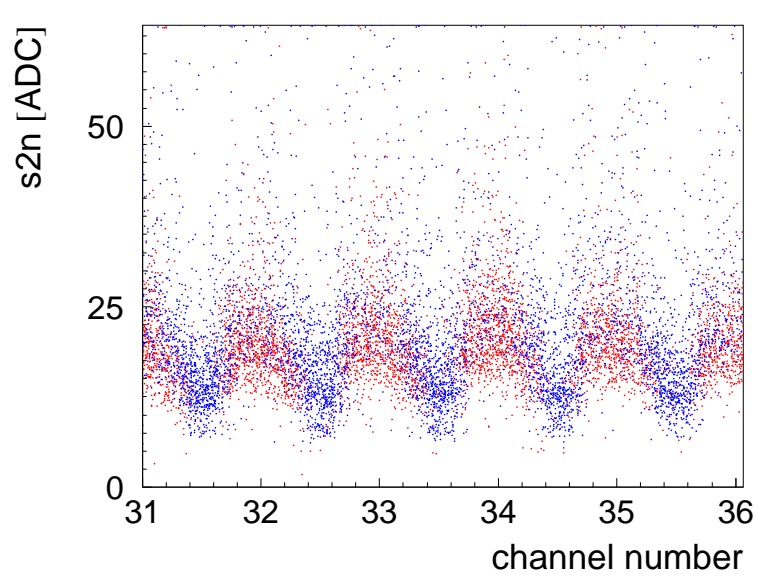

$90 \mathrm{~V}$ biasing voltage at room temperature

resolution $\sim 50 \mu \mathrm{m}$
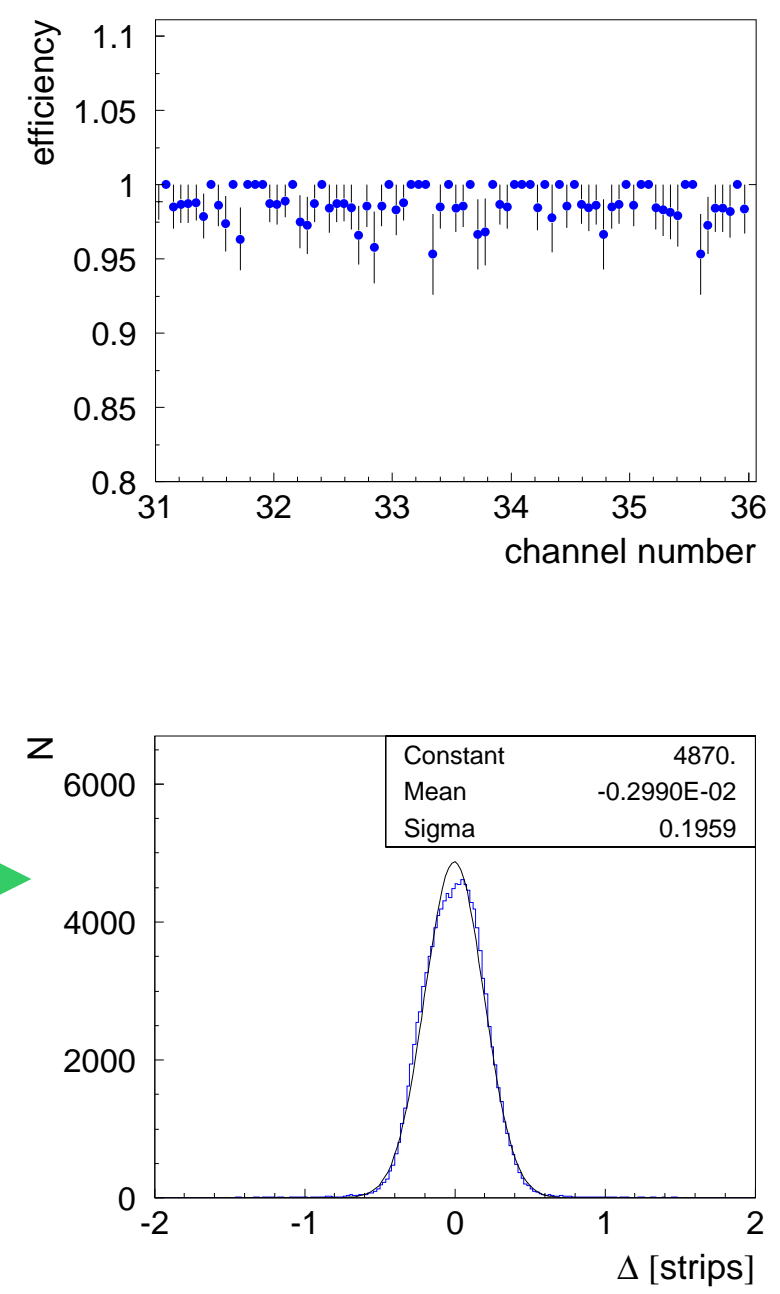


\section{Priliminary Testbeam Results for Long Sensor Ladder}

$80 \mathrm{~V}$ biasing voltage

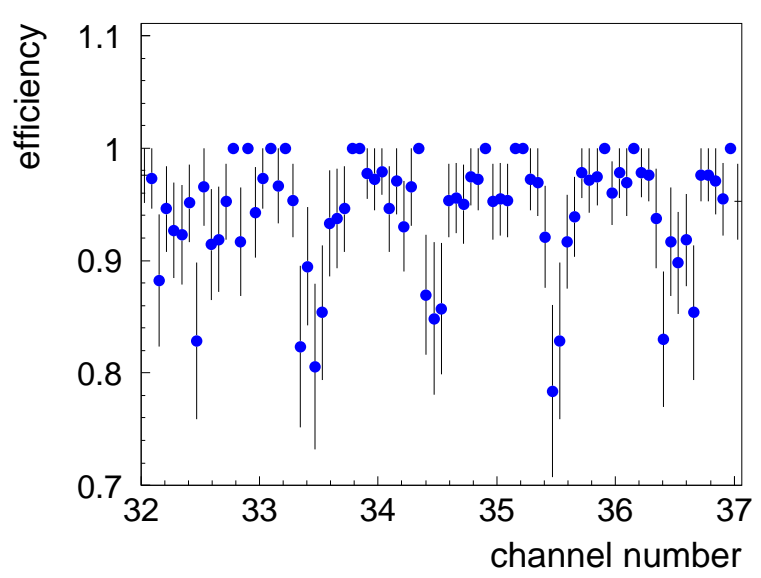

$100 \mathrm{~V}$ biasing voltage

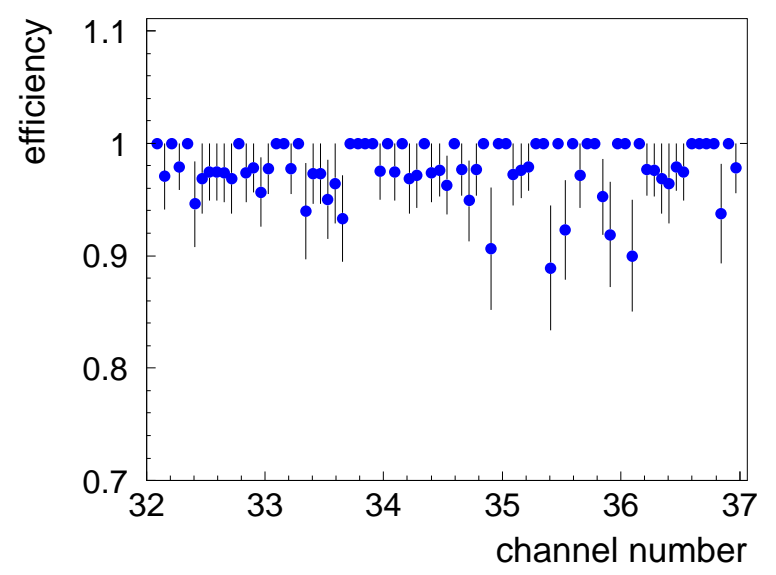




\section{Frontend Electronics and Link}

(2 Beetle readout chip specifically developed for $\mathrm{LHCb}$ :

128 channels, analogue pipeline and $40 \mathrm{MHz}$ readout speed

( each ladder equipped with 3 Beetle
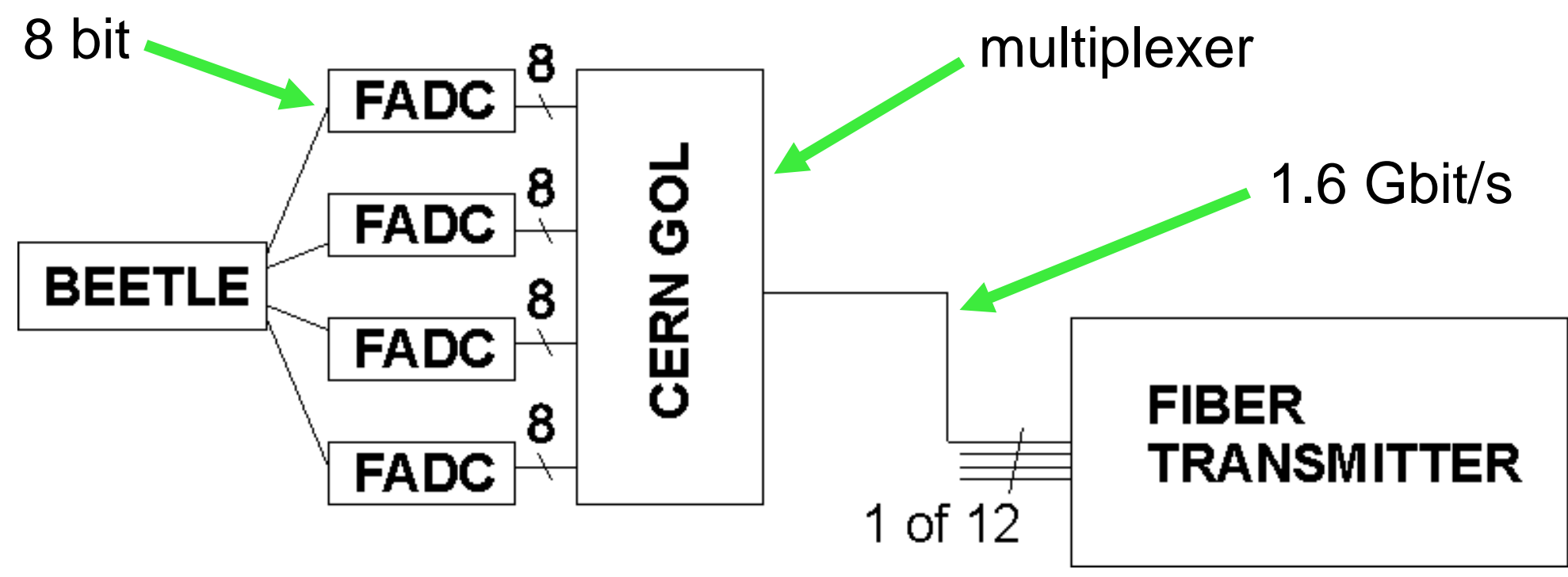

test set-up is under construction

ODE 


\section{Next Steps}

( BEETLE readout chip availble now design board

(2) detector specs reviewed order 6" sensors

(2 station mechanics including cooling building fullsized prototype

C optical link build up test setup

TDR to be submitted end of this year 